\author{
Marquette University \\ e-Publications@Marquette
}

College of Nursing Faculty Research and

Publications

Nursing, College of

8-2012

\title{
Parenting and Feeding Behaviors Associated With School-Aged African American and White Children
}

Michele L. Polfuss

Medical College of Wisconsin

Marilyn Frenn

Marquette University College of Nursing, marilyn.frenn@marquette.edu

Follow this and additional works at: https://epublications.marquette.edu/nursing_fac

Part of the Nursing Commons

\section{Recommended Citation}

Polfuss, Michele L. and Frenn, Marilyn, "Parenting and Feeding Behaviors Associated With School-Aged African American and White Children" (2012). College of Nursing Faculty Research and Publications. 126. https://epublications.marquette.edu/nursing_fac/126 
Marquette University

e-Publications@Marquette

\title{
Nursing Faculty Research and Publications/College of Nursing
}

This paper is NOT THE PUBLISHED VERSION; but the author's final, peer-reviewed manuscript. The published version may be accessed by following the link in the citation below.

Western Journal of Nursing Research, Vol. 34, No. 5 (August 1, 2012): 677-696. DOI. This article is (C) SAGE Publications and permission has been granted for this version to appear in ePublications@Marquette. SAGE Publications does not grant permission for this article to be further copied/distributed or hosted elsewhere without the express permission from SAGE Publications].

\section{Parenting and Feeding Behaviors Associated with School-Aged African American and White Children}

Michele Lynn Polfuss

Medical College of Wisconsin, Milwaukee, WI

Marilyn Frenn

Marquette University, Milwaukee, WI

\begin{abstract}
Pediatric obesity is multifactorial and difficult to treat. Parenting and feeding behaviors have been shown to influence a child's weight status. Most prior studies have focused on preschool-aged White children. Additional complicating factors include parents' inability to accurately identify their child's abnormal weight status. Parenting and feeding behaviors used by 176 African American and White parents of school-age children were examined. Assessment included (a) identifying what behaviors were reported when parent expressed concern with child's weight and (b) the relationship of these behaviors on child's body mass index percentile (BMI\%), considering ethnicity, socioeconomic status (SES), and parent's body mass index (BMI). Findings included African American parents and parents
\end{abstract}


concerned about their child's weight exhibited increased controlling/authoritarian parenting and feeding behaviors. Parents were able to accurately identify their child's weight status. Parenting and feeding behaviors played a significant role in the children's BMI\% even when controlling for ethnicity, SES, and parent's BMI.

\section{Keywords}

diet and eating, health behavior/symptom focus, parenting/families, population focus, pediatrics, Black, adolescence

According to the 2007-2008 National Health and Nutrition Examination Survey (NHANES), 31.7\% of children and adolescents are considered overweight or obese (Ogden, Carroll, Curtin, Lamb, \& Flegal, 2010). Overweight is defined as body mass index percentile (BMI\%) $\geq 85$ and obesity as BMI\% $\geq 95$ for age and gender (Ogden et al., 2010). Groups disproportionately affected include African Americans and those with a lower socioeconomic status (SES; Centers for Disease Control and Prevention, 2009; Singh, Siahpush, \& Kogan, 2010; Wang \& Beydoun, 2007). The importance of the parent's role in moderating the child's environment cannot be underestimated.

Previous research has examined how parenting and feeding behaviors affect a child's weight status, but these studies have mainly focused on preschool-aged White children (Birch \& Fisher, 2000; Fisher \& Birch, 1999; Rhee, Lumeng, Appugliese, Kaciroti, \& Bradley, 2006; Wake, Nicholson, Hardy, \& Smith, 2007). Unfortunately, the obesity epidemic continues across all ages and limited research has addressed the school age and adolescent child within the parenting and feeding-behavior context. An additional concern includes findings that many parents are unable to accurately identify when their child has an abnormal weight status (Baughcum, Chamberlin, Deeks, Powers, \& Whitaker, 2000; Doolen, Alpert, \& Miller, 2009; Eckstein et al., 2006), which can complicate attempts at treatment of obesity. This study examined parenting and feeding behaviors that are used by African American and White parents of children between the ages of 9 to 15 years old when the parent identifies the child's weight as a concern. Further assessment included whether the parent's concern was appropriate and how the ethnicity, SES, and/or the parent's weight affect these relationships.

The family or the parents are often thought of as the focal point for interventions to address pediatric obesity (Golan \& Crow, 2004; Golan, Kaufman, \& Shahar, 2006). The act of parenting is a process of complex two-way interactions that occur between the child and the parent (Luther, 2007). Seminal work was performed by Diana Baumrind who examined parenting through the context of amount of responsiveness, sensitivity, and demandingness that parents exert on the child (Baumrind, 1966, 2005). Through her work, three models of parenting emerged: permissive, authoritarian, and authoritative (Baumrind, 1966). Baumrind's work was further elaborated by Maccoby and Martin (1983), which led to the inclusion of a fourth parenting style, neglectful.

As research within the parenting context continued, parenting behaviors were deconstructed to three aspects: (a) the level of acceptance versus the level of rejection, (b) the level of psychological control versus psychological autonomy, and (c) the level of firm control versus the level of lax control (Barber, Stolz, \& Olsen, 2005; Dornbusch, Ritter, Leiderman, Roberts, \& Fraleigh, 1987). Emphasis has focused 
on the authoritarian and authoritative parenting styles. Authoritative parents use a higher level of acceptance, exert firm control but will not overwhelm the child with restrictions, and allow for psychological autonomy. Children of authoritative parents emerge as socially responsible and more independent than children raised by parents using the other typologies (Baumrind, 1966; Dornbusch et al., 1987). Authoritative parenting is more prevalent among European-American and middle-class families, with these children more likely to have these parenting practices echoed in their neighborhoods and social circles (Steinberg, Lamborn, Darling, Mounts, \& Dornbusch, 1994). In a brief review of six studies that examined parenting styles and obesity, Luther (2007) summarized that the authoritarian, permissive, and neglectful parenting styles were associated with an increased likelihood of childhood obesity. During an intervention study that examined childhood-obesity treatment of school-age children through teaching of the parents, there was a greater weight reduction in the children of the parents who increased their use of authoritative parenting behaviors (Golan et al., 2006).

Is the authoritative style of parenting always superior across different ethnic backgrounds, cultures, and socioeconomic classes? It has been suggested that in certain situations, a parent who uses a strict and more vigilant level of control (authoritarian parenting) may benefit the child (Steinberg et al., 1994). The example commonly provided includes a child from an economically disadvantaged background, who may live in an unsafe environment. These circumstances may necessitate a more authoritarian approach for safety purposes (Steinberg et al., 1994). Unfortunately, when a more psychologically controlling and firm parenting style is used (authoritarian parenting), what is often found is that the child is limited from developing as an individual (Schaefer, 1965). As children age and begin to establish their own identity, they will begin to experiment with choices they control, including nutritional intake (Kaur et al., 2006). African American families have been shown to use increased authoritarian and neglectful parenting styles when feeding preschool-aged children (Hughes, Power, Orlet Fisher, Mueller, \& Nicklas, 2005), but this has not been well examined with parents of older children.

When linking parenting behavior with childhood obesity, feeding practices utilized by the parent have also been examined. Restrictive feeding practices and pressuring the child to eat have been associated with overeating and poorer self-regulation in preschool-age children (Eneli, Crum, \& Tylka, 2008; Savage, Fisher, \& Birch, 2007). Excessive parental control and pressure to eat may influence dietary intake and disrupt the child's behavioral control of food intake (Savage et al., 2007). Savage et al. (2007) noted that parents with more controlling or authoritarian feeding practices have fewer fruits and vegetables available in their homes and their children consume less of these food groups. The authoritarian parenting style has also been associated with an increased consumption of sweetened beverages, salty snacks, and decreased milk intake (Demory-Luce et al., 2004).

Authoritative feeding practices have been positively associated with increased consumption of dairy and vegetables (Moens, Braet, \& Soetens, 2007). The authoritative feeding practice would be when the parents are highly responsive to their child's eating cues and behaviors allowing them to participate in the feeding process, yet set limits and have clear expectations regarding the child's needs (Savage et al., 2007). Similar to general parenting, this is thought of as the superior feeding style that promotes appropriate growth and nutrition (Moens et al., 2007; Rhee et al., 2006). Although an authoritarian 
style of parenting can be seen as a benefit in some cultures related to safety (Steinberg et al., 1994), there have not been any associated benefits found when utilizing these parenting behaviors within the feeding context.

Differences have been found among ethnic groups when assessing feeding behaviors used. Similar to parenting styles, African American families have been shown to use increased authoritarian feeding behaviors such as higher restriction and monitoring of food intake of their children (Eneli et al., 2008; Hughes et al., 2005). Specific examples of authoritarian feeding behaviors include restricting access to or controlling types and amount of food, specifically foods high in fat and sugar, rewarding or punishing with food, and pressuring the child to eat with a "clean your plate" mentality regardless of hunger (Birch \& Fisher, 2000; Fisher \& Birch, 1999; Hughes et al., 2005).

Some researchers have proposed that parenting behaviors are not fixed and that a parent may change their parenting behavior based on the specific problem being dealt with, depending on their level of concern and/or level of constraint in that problem area (Costanzo \& Woody, 1985). Costanzo and Woody (1985) theorized that the parent's own individual values and perceptions of the long-term consequences, thought to be associated within that particular domain (e.g., obesity), would determine their level of concern. Using this approach with parenting behaviors and feeding, it would be important to know the level of concern that a parent attributed to the child's weight to help understand the parenting behavior that they use with feeding. Understanding that the parenting behaviors may be different or heightened within this context, education and possible interventions can be delivered with a higher likelihood of success.

The majority of studies that have assessed feeding behaviors, parenting behaviors, and children's weight have been performed with preschool or younger children of White descent (Fisher \& Birch, 1999; Rhee et al., 2006; Wake et al., 2007). The common assumption is that this would be an optimal time frame that would allow for easier changes to be implemented as habits are less engrained. Because of the vast diversity among developmental stages of children and ethnic groups, the findings from these studies cannot be generalized for use with other age groups. This leaves a large population of older children who cannot benefit from prior research.

Although less dependence on parents occurs with older children, parents still play a major role in the school-age child's life (Van der Horst et al., 2007). Their parenting and feeding behaviors will affect the child's day-to-day life through mechanisms such as buying groceries, preparation of meals, and discipline techniques to name a few. Although habits may be more engrained as a child ages, changes can be made (Foster et al., 2010). To address the current obesity epidemic that occurs across all ages, it is imperative to understand how parenting and feeding behaviors relate to obesity in the older child. This will allow another avenue of interventions that will aim to stabilize or decrease obesity rates in our older children and prevent them from becoming obese adults.

Parents have been recognized as key players in treating pediatric obesity (Golan \& Crow, 2004). Unfortunately, if a parent does not recognize that their child has a problem with excess body weight, there is a decreased chance that they will assist in making changes that will result in weight loss for the child. In a metasynthesis of 15 studies that examined parental perception of their child's weight, it was found that the majority of parents failed to accurately perceive whether their child was overweight or 
obese (Doolen et al., 2009). Factors that have been associated with an inaccurate perception of child's weight includes parents: (a) with lower education (Baughcum et al., 2000; Goodman, Hinden, \& Khandelwai, 2000); (b) of African American descent, where a larger body size may have greater acceptance (Baughcum et al., 2000; Young-Hyman, Herman, Scott, \& Schlundt, 2000); and (c) of sons (Doolen et al., 2009; Maynard, Galuska, Blanck, \& Serdula, 2003). Conversely, in a study of 114 African American and Hispanic dyads, O'Neil et al. (2010) found that 99 (86.8\%) of the parents correctly identified that their child was overweight. The need for further research that examines parenting and feeding behaviors with diverse ethnic samples has been documented (Wardle \& Carnell, 2006).

Being able to appropriately acknowledge that a child is overweight may be the first step in engaging a parent to want to make changes. Studies have shown that even if a parent acknowledges that their child is overweight they are not always concerned (Baughcum et al., 2000; Doolen et al., 2009; Eckstein et al., 2006), which can negatively impact likelihood of change occurring. Eckstein et al. (2006) found that parents of children more than 6 years of age were more likely to recognize their child's overweight status.

The conceptual framework for this study has the underlying belief that general parenting styles are consistently present, but parenting behaviors within that style will increase or decrease based on the situation or domain. With each parenting interaction, an iterative process occurs where a parent actively observes a situation and frames the situation with their internal values and assessment of short- and long-term consequences for the child associated with the situation. Based on this process, parents will react with a level of acceptance/rejection, psychological control/psychological autonomy, and firm/lax control, which is an aspect of their general parenting style but may be exaggerated in a domain where the parent has increased concern. For parents with concern about their child's weight, this will translate to specific feeding behaviors used. These interactions over time will determine the child's outcome in this domain. Children who are parented with higher levels of acceptance, psychological autonomy, and firm control will have improved weight control and a healthier body mass index (BMI). Children who are parented with higher levels of rejection, psychological control, and lax control will experience poor weight control and an unhealthy BMI.

\section{Purpose}

Specific research questions were as follows: (a) What feeding behaviors and parenting behaviors are reported when the parent identifies an increased concern about the school-age child's weight status? (b) Do these associations vary between ethnic groups? (c) Is parental concern about their child's weight accurately based on an overweight or obese BMI\%? (d) Is the relationship between parenting, feeding behaviors, and a school-age child's BMI\% influenced by ethnicity, SES, and/or parent's BMI?

\section{Method}

A cross-sectional design was used with a convenience sample of 176 parent/child African American and White dyads from a Midwestern state. Power analysis performed with a beta of .20, two-sided alpha of .05 and estimated effect size of 0.30 identified the goal sample size of 85 parent/child dyads for each ethnic group-African American and White. 


\section{Setting and Sample}

Participants were recruited from a variety of locations within one Midwestern state. Study sites included a private pediatric clinic, two boys and girls clubs, an inner-city, nurse-practitioner-run clinic, a pediatric-specialty clinic, and an inner-city, university-based dental clinic.

Inclusion criteria required that the participants were (a) alert and oriented, (b) able to speak and read the English language, (c) willing to have a height and weight performed, and (d) either African American or White. Child participants needed to be between the ages of 9 and 15 years old and the parent needed to be a biological parent of the child who lived with that child at least $50 \%$ of the time.

Only one child per family was included into the study. If more than one child was interested in participating in the study, the child with the birth date closest to January 1st was included. If more than one parent wanted to participate, the other parent was allowed to participate but there was no additional reimbursement. During data collection, if participants had been unable to communicate or had cognitive impairment that prohibited them from completing the study, their data would have been excluded.

\section{Measures}

\section{Demographics}

A general demographic form was utilized. Biological parental status, gender and age of child and parent, family-income level, educational level, and qualification for free- or reduced-lunch status at school were included.

\section{Parenting behaviors}

Parents completed the 30-item Child Report of Parenting Behavior Inventory-Parent Version (CRPBI; Schludermann \& Schludermann, 1988). The CRPBI assesses three dimensions of parenting: Acceptance Versus Rejection, Psychological Control Versus Psychological Autonomy, and Firm Control Versus Lax Control (Schludermann \& Schludermann, 1988). Each of the three subscales had 10 items that provided a range of 10 to 30 points. Internal consistency of the 30-item CRPBI was adequate with Cronbach's alpha scores ranging from .63 to .75 (Schludermann \& Schludermann, 1988). Additional studies with the CRPBI have found acceptable reliability and validity (Butler, Skinner, Gelfand, Berg, \& Wiebe, 2007; Greenley, Holmbeck, \& Rose, 2006).

Within the current study, internal consistency was adequate with Cronbach's alpha $=.67$ (Firm Control Versus Lax Control), .82 (Psychological Control Versus Psychological Autonomy), and .81 (Acceptance Versus Rejection). Internal consistency varied when the instrument was assessed for each ethnic group. For the White population, internal consistency was .45 (Firm Control Versus Lax Control), .83 (Psychological Control Versus Psychological Autonomy), and .84 (Acceptance Versus Rejection). For the African American population, internal consistency was .72 (Firm Control Versus Lax Control), .86 (Psychological Control Versus Psychological Autonomy), and .78 (Acceptance Versus Rejection).

Parents responded to questions across the three domains of acceptance (e.g., "smiles at my child very often"), psychological control (e.g., "is less friendly toward my child if she or he does not see things my way"), and firm versus lax control (e.g., "insists that my child must do exactly as she or he is told") by choosing if they were "like," "somewhat like," or "not like" each of the items listed. Higher scores 
within each subscale reflected increased acceptance, psychological control, and firmness. See Table 1 for additional instrument information.

Table 1. Instrument Descriptive Statistics

\begin{tabular}{|c|c|c|}
\hline Subscales & $M(S D)$ & $95 \% \mathrm{Cl}$ \\
\hline \multirow{3}{*}{$\begin{array}{l}\text { Child report of Parent Behavior Inventory - Parent Version } \\
\text { Acceptance Versus Rejection }\end{array}$} & W $27.50(2.78)$ & {$[-1.76,0.62]$} \\
\hline & C $27.71(2.76)$ & \\
\hline & AA $27.29(2.80)$ & \\
\hline \multirow[t]{3}{*}{ Psychological Control Versus Psychological Autonomy } & W $16.00(4.38)$ & {$[-4.63,-1.97]$} \\
\hline & C $14.47(3.55)$ & \\
\hline & AA 17.56 (4.61) & \\
\hline \multirow[t]{3}{*}{ Firm Versus Lax } & W $16.62(4.42)$ & {$[-1.97,-0.25]$} \\
\hline & C $15.66(2.12)$ & \\
\hline & AA 17.60 (3.35) & \\
\hline \multirow[t]{3}{*}{ Child Feeding Questionnaire Concern of child overweight } & W 8.67(4.42) & {$[-1.03,-0.17]$} \\
\hline & C $7.78(4.35)$ & \\
\hline & AA $9.58(4.34)$ & \\
\hline \multirow[t]{3}{*}{ Restriction } & W $25.21(7.97)$ & {$[-0.75,-0.17]$} \\
\hline & C23.39 (8.05) & \\
\hline & AA 27.07 (7.48) & \\
\hline \multirow[t]{3}{*}{ Pressure to eat } & W $10.08(4.38)$ & {$[-1.06,-0.44]$} \\
\hline & C 8.60 (3.99) & \\
\hline & AA $11.60(4.27)$ & \\
\hline \multirow[t]{3}{*}{ Monitoring } & W 10.55 (3.20) & {$[-0.53,0.10]$} \\
\hline & C $10.22(3.46)$ & \\
\hline & AA 10.87 (2.90) & \\
\hline
\end{tabular}

Note: $\mathrm{Cl}=$ confidence interval; $\mathrm{W}=$ whole sample $(\mathrm{N}=176)$; $\mathrm{C}=$ White $(\mathrm{n}=89)$;

$A A=$ African American ( $n=87)$. White coded as 1, African American coded as 2.

\section{Parent's perception and concerns regarding child obesity}

The Child Feeding Questionnaire (CFQ) has a total of 31 items that are answered on a 5-point Likerttype scale (Birch et al., 2001). A total of seven subscales were measured including the parent's responsibility for feeding the child, parent's monitoring of the child's nutritional intake, parent's restricting the nutritional intake, parental practices of encouraging food intake, perceived child weight, perceived parent self-weight, and parent's concern about their child's weight (Birch et al., 2001; Kaur et al., 2006). Concern about the child's weight was defined as the parent having concern that their child was eating too much when the parent was not around, would have to diet to maintain a desirable weight, and would become overweight (Kaur et al., 2006). The number of items within the subscales reported and range of points are as follows: concern of child overweight consisted of three items providing a range of 3 to 15 points, restriction had eight items and a range of 8 to 40 points, pressure to eat had four items and a range of 4 to 20 points, and monitoring had three items with a range of 3 to 15 points. Internal consistency was acceptable in prior studies with scores ranging from Cronbach's alpha of .70 for pressure to eat to .92 for monitoring of food (Birch et al., 2001). In the present study, 
Cronbach's alpha scores were found to be acceptable with pressure to eat (.75), restriction (.86), concerns about child overweight (.88), and monitoring (.90). The instrument was reliable for both ethnic groups with the following internal consistencies: pressure to eat (White $=.73$, African American $=.72$ ), concern about child's weight (White $=.89$, African American $=.86$ ), restriction (White $=.88$, African American $=.85$ ), and monitoring (White $=.93$, African American $=.86$ ). See Table 1 for additional instrument information.

\section{Anthropometrics}

Weight status was obtained by a trained health care provider who measured the individual's height and weight with shoes removed. Seca 869 portable medical grade scale and Seca 214 portable stadiometer were used for nonmedical sites. Calibrated medical grade Seca digital scale and wallmounted stadiometer were used for medical sites. Height and weight measurements were converted to a BMI for adults and a BMI\% for the children. BMI\% scores standardized for age and sex according to the national norms were obtained from Centers for Disease Control and Prevention at http://www.cdc.gov/healthyweight/assessing/bmi/.

\section{Procedures}

Participants were recruited through flyers and personal contact at the study settings. Written consent and assents were obtained from the parent and the child prior to participating within the study. Approval for the study was granted through the internal review boards of a midwestern children's hospital and university.

The parent was asked to complete a demographic-information sheet and two questionnaires and the child was asked to complete one questionnaire. Completion of paperwork was done in a private area. Parent and child completed forms independently. A research assistant or the principle investigator offered to assist the children with reading of questions and was available to answer any questions of participants. Once paperwork was completed, shoes and outer clothing were removed. The standing height and weight were performed.

A US\$10.00 gift certificate to a local department store was offered to each individual (parent and child) who completed the questionnaires and had a height and weight performed. Data collection required 30 min including the consenting and assenting process. Compensation was chosen based on literature stating that financial incentives have been shown to increase participation rates and that an average of US\$10.00 per hour of work has been previously utilized (Rudy, Estok, Kerr, \& Menzel, 1994; Steinke, 2004).

\section{Analysis}

Data were analyzed using SPSS Version 18.0. All $p$ values were two-tailed, with a $p<.05$ considered statistically significant. Descriptive statistics and comparison of means were calculated. Collinearity diagnostics of tolerance and variance inflation factors (VIF) were found to be within acceptable ranges.

For Research Question 1, a general linear model was used. The independent variables were as follows: (a) the concern that the parent had about the child's weight recoded into a dichotomous variable at the mean and (b) ethnicity. The dependent variables were as follows: (a) Acceptance Versus Rejection, (b) Psychological Control Versus Psychological Autonomy, (c) Firm Control Versus Lax Control, (d) 
restriction of food, (e) pressure to eat, and ( $f$ ) monitoring of food. Eta square was calculated as the sum of squares between, divided by total sum of squares, with .01 considered a small effect, .06 a medium effect, and .14 a large effect (Polit \& Beck, 2008).

For Research Question 2, Pearson's product-moment correlation coefficient analysis was used. For Research Question 3, a sequential regression analysis was performed. The dependent variable was the child's BMI\%. The first block of variables included the following: (a) ethnicity, (b) SES derived from family income, and (c) parent BMI. The second block of variables were the following: (a) Acceptance Versus Rejection scale, (b) Psychological Control Versus Psychological Autonomy, (c) Firm Control Versus Lax Control, (d) restriction of food, (e) pressure to eat, and (f) monitoring of food.

\section{Results}

Of the dyads, 89 (50.6\%) described themselves as White and 87 (49.4\%) as African American descent. Children's ages ranged between 9 and 15 years (mean age 11.6 years, $S D=1.95$ ). Parents' ages ranged from 23 to 59 (mean age 38.2 years, $S D=7.00$ ). Mothers made up the majority of the parents with 148 (84.1\%) participating versus 28 (15.9\%) fathers. The children who completed the surveys were made up of 92 males (52.3\%) and 84 females (47.7\%). Ninety-three $(52.8 \%)$ of the parents were working fulltime, 37 (21\%) were working $20 \mathrm{hr}$ or less, 34 (19.3\%) were not working, and an additional 6 (3.4\%) were disabled. Annual family income was broken down by US\$0 to US\$25,000 (40.3\%), US\$26,000 to $\$ 50,000$ (31.8\%), US\$51,000 to US\$75,000 (13.6\%), US\$76,000 to US\$100,000 (9.1\%), and $>$ US\$100,000 (5.1\%). One hundred and six (60.2\%) of the children qualified for free or reduced lunch. Table 2 conveys descriptive statistics.

- Research Question 1: (a) What feeding behaviors and parenting behaviors are reported when the parent identifies an increased concern about the school-age child's weight status? (b) Do these associations vary between ethnic groups?

Table 2. Parent/Child Anthropometrics

\begin{tabular}{|l|l|l|l|}
\hline Anthropometric & $n$ & $M(S D)$ & $95 \% \mathrm{Cl}$ \\
\hline Parent BMI & 176 & $32.15(8.12)$ & {$[-5.00,-0.22]$} \\
\hline White & 89 & $30.86(7.60)$ & \\
\hline African American & 87 & $33.47(8.46)$ & \\
\hline Child BMI & 176 & $23.21(7.53)$ & {$[-3.26,1.22]$} \\
\hline White & 89 & $22.71(7.88)$ & \\
\hline African American & 87 & $23.73(7.16)$ & \\
\hline Child BMI percentile & 176 & $74.76(26.90)$ & {$[-17.48,-1.72]$} \\
\hline White & 89 & $70.02(29.07)$ & \\
\hline African American & 87 & $79.62(23.69)$ & \\
\hline
\end{tabular}

Note: $\mathrm{Cl}=$ confidence interval; $\mathrm{BMI}=$ body mass index.

As shown in Table 3, parents identifying a high concern about their child's weight used increased rejection in their parenting (small effect) and more restriction of food (large effect) and practiced more monitoring of food (medium effect) than those with low concern. African American as compared with White parents reported using more psychological control (medium effect), firm parenting practices 
(small effect), restriction of food (small effect), and pressure to eat (medium effect). The interaction of concern and race was significant only for pressure to eat (small effect).

- Research Question 2: Is parental concern about their child's weight accurately based on an overweight or obese BMI\%?

Table 3. Significant Effects of Parent Concern and Race on Parenting and Feeding Behaviors

\begin{tabular}{|c|c|c|c|c|}
\hline & $\mathrm{M}(\mathrm{SD})$ & $\mathrm{F} \mathrm{df}(1,175)$ & $p$ & $\eta 2$ \\
\hline Concern about child overweight & & 5.49 & .02 & .03 \\
\hline \multicolumn{5}{|l|}{ Parent acceptance versus rejection ${ }^{a}$} \\
\hline Low & $28.09(2.25)$ & & & \\
\hline High & $27.05(3.06)$ & & & \\
\hline Restriction of food & & 35.15 & .00 & .16 \\
\hline Low & $2.64(0.99)$ & & & \\
\hline High & $3.53(0.82)$ & & & \\
\hline Monitoring of food & & 10.66 & .001 & .06 \\
\hline Low & $3.20(1.25)$ & & & \\
\hline High & $3.76(0.83)$ & & & \\
\hline \multicolumn{5}{|l|}{ Race } \\
\hline Parent psychological control versus autonomy & & 18.09 & .00 & .09 \\
\hline AA & $17.56(4.61)$ & & & \\
\hline $\mathrm{C}$ & $14.47(3.55)$ & & & \\
\hline Parent firm versus lax ${ }^{\mathrm{C}}$ & & 4.02 & .05 & .02 \\
\hline AA & $23.44(2.78)$ & & & \\
\hline $\mathrm{C}$ & $22.67(2.77)$ & & & \\
\hline Restriction of food & & 3.92 & .05 & .02 \\
\hline AA & $3.38(0.94)$ & & & \\
\hline C & $2.92(1.01)$ & & & \\
\hline Pressure to eat & & 20.81 & .00 & .10 \\
\hline $\mathrm{AA}$ & $2.90(1.07)$ & & & \\
\hline $\mathrm{C}$ & $2.15(1.00)$ & & & \\
\hline \multicolumn{5}{|l|}{ Interaction } \\
\hline \multicolumn{5}{|l|}{ Pressure to eat } \\
\hline Low concern & & 5.69 & .02 & .03 \\
\hline AA & $2.73(0.98)$ & & & \\
\hline $\mathrm{C}$ & $2.39(0.98)$ & & & \\
\hline \multicolumn{5}{|l|}{ High concern } \\
\hline $\mathrm{AA}$ & $2.98(1.11)$ & & & \\
\hline $\mathrm{C}$ & $1.87(0.96)$ & & & \\
\hline
\end{tabular}

Note: $A A=$ African American coded as 2; $C=$ White coded as 1 . Significant effects using general linear model analysis. Low concern coded as 1 . High concern coded as 2 .

aHigher score indicates higher parent acceptance, lower score indicates higher parent rejection.

bHigher score indicates higher parent psychological control, lower score indicates higher parent autonomy. cHigher score indicates higher parent firmness, lower score indicates higher parent laxness. 
Of the 176 parents, 86 expressed that they were concerned about their child's weight. Concern was specific to the child eating too much, having to diet, or becoming overweight. The majority of the parents expressing concern regarding their child's weight were appropriately concerned with 50 (58.1\%) of these children being clinically obese (BMI > 95\% for age and gender) and 19 (22.1\%) being clinically overweight (BMI between the $85 \%$ and $95 \%$ for age and gender). However, 16 (18.6\%) of these children were actually within the normal-weight category (BMI $>5 \%$ and $<85 \%$ for age and gender) and 1 (1.2\%) was in the underweight category (BMI < 5\% for age and gender).

- Research Question 3: Is the relationship between parenting, feeding behaviors, and a schoolage child's BMI\% influenced by ethnicity, SES, and/or parent's BMI?

Sequential regression analysis was performed to assess the relationship between parenting/feeding behaviors and the child's weight while controlling for ethnicity, statuses, and parent's BMI. In the first model that was comprised of ethnicity, family income, and parent BMI, the model explained $6 \%$ of the variance of the child's BMI\%; $R^{2}=.06, F(3,172)=3.47, p=.02$. After the parenting behaviors were added, the model explained $51 \%$ of the variance of the child's BMI\% or an additional $45 \%$ of the variance after controlling for ethnicity, SES, and parent $\mathrm{BMI} ; R^{2}=.51, F(9,166)=6.40, p=<.001$.

In Model 1, only the parent BMI was a significant contributor. In Model 2, when adding the parenting behaviors, the parent $\mathrm{BMI}$ did not remain as a unique contributor, but rejection of the child, restriction of food, and pressure to eat were significant. See Table 4 for additional information.

Table 4. Sequential Multiple Regression Analysis Predicting Effect of Parenting Behaviors on Child's BMI Percentile

\begin{tabular}{|l|l|l|l|}
\hline & Model 1 & Model 2 & \\
\hline Variable & $\mathrm{B}$ & $\mathrm{B}$ & $95 \% \mathrm{Cl}$ \\
\hline Constant & $46.77^{* * *}$ & $100.62^{* * *}$ & {$[44.44,156.81]$} \\
\hline Ethnicity & 7.90 & 8.12 & {$[-0.28,16.53]$} \\
\hline Annual Family Income & -0.02 & -1.82 & {$[0.30,-5.25]$} \\
\hline Parent BMI & $0.53^{*}$ & 0.37 & {$[-0.08,0.82]$} \\
\hline Acceptance vs. Rejection & & $-1.76^{* *}$ & {$[-3.10,-0.43]$} \\
\hline Psychological Control vs. Psychological Autonomy & & -0.67 & {$[-0.29,1.63]$} \\
\hline Firm vs. Lax & & -0.50 & {$[-1.82,0.81]$} \\
\hline Restriction & & $-6.80^{* *}$ & {$[2.16,11.43]$} \\
\hline Pressure & & $-9.43^{* * *}$ & {$[-13.22,-5.63]$} \\
\hline Monitoring & & -1.62 & {$[-2.59,5.83]$} \\
\hline
\end{tabular}

Note: $\mathrm{N}=176 . \mathrm{Cl}=$ confidence interval; $\mathrm{BMI}=$ body mass index.

$* p<.05 . * * p<.01 . * * * p<.001$.

\section{Discussion}

When the parent of the 9- to 15-year-old child identified that they were concerned with their child's weight, associated findings for both White and African American parents included increased rejecting of the child and using authoritarian feeding behaviors such as restriction of food and monitoring of their child's feeding when compared with the parents who were not concerned about their child's 
weight. Specifically, the parents restricted access or portion sizes of foods and monitored consumption of energy-dense food.

This supports previous findings with younger children that higher levels of concern will be associated with exerting increased control over feeding (Birch \& Fisher, 2000; Musher-Eizenman, Holub, Hauser, \& Young, 2007). Restriction of food similarly has been shown to counteract the parent's attempt to have a child eat healthier (Fisher \& Birch, 1999; Musher-Eizenman et al., 2007). When sweets or junk food are used as a reward for eating healthy foods or when favorite nonnutritious food items are restricted from the child, there is a rebound effect. The child ends up not liking the healthy food they were coerced to eat or they seek out the restricted items and eat in higher amounts (Birch \& Fisher, 2000; Fisher \& Birch, 1999; Klesges, Stein, Eck, Isbell, \& Klesges, 1991; Musher-Eizenman et al., 2007). It is uncertain if parents are aware of these behaviors or understand how these behaviors may have a negative effect on their child. This is an opportunity for discussion and education.

When comparing African American and White parents who identified that they were concerned with their child's weight, African American parents demonstrated increased pressuring of the child to eat. It seems contradictory that a parent who is concerned about their child's weight would pressure the child to eat but it is not certain which behavior occurred first, the concern about the weight or the pressuring the child to eat. The possible explanation of this is that the child became overweight through the continued pressure to eat or clean the plate mentality.

With the current obesity epidemic, it is important for parents to be concerned about their child's weight, but we must be careful that parents are accurate in their concern. From the study, it was noted that $80.2 \%$ of parents who expressed concern were appropriate as their child was above the recommended weight for their age and gender, supporting the previous findings that parents of older (Eckstein et al., 2006) and African American or Hispanic (O'Neil et al., 2010) children have a better perception of their child's weight. Unfortunately, $19.8 \%$ were inaccurate with their concerns, which may result in inappropriate parenting or feeding practices for children who are normal or underweight.

Differences were found between the ethnic groups in parenting styles and feeding behaviors. Specifically, for parenting styles, African American parents demonstrated increased psychological control and firmness. In terms of feeding behaviors, African American parents used increased restriction of food and pressuring the child to eat when compared with White parents. This supports previous literature showing that African Americans use authoritarian parenting and feeding behaviors (Birch \& Fisher, 2000; Eneli et al., 2008; Hughes et al., 2005; Wake et al., 2007), but not neglectful. Authoritarian feeding behaviors can reduce the child's ability to regulate what and how much they eat based on hunger and satiety, and thereby have the ability to contribute to higher BMI\% (Eneli et al., 2008). Acknowledging that the prevalence of pediatric obesity is increased in the African American population, this finding should be acknowledged and further studied.

An additional difference found between the ethnic groups was how reliable the instrument was when used separately for each ethnic group. In this study, the Firmness Versus Laxness subscale had a poor internal-consistency score when used for the White participants alone. This prohibited the use of the Firmness subscale to be used as a reliable indicator for the White population. These findings were unexpected as the instrument was previously validated with a White sample with acceptable reliability 
scores. The other subscales were reliable for use with the entire sample and within each ethnic group separately. This finding documents the importance of assessing reliability within a sample as differences by ethnicity can be found.

Ethnicity, family income, and parent BMI had surprisingly little influence on the child's weight. Parenting and feeding behaviors had a much larger effect on the child's weight status. This contradicts extensive previous research that has concluded that the genetic influences on BMI are substantial (Stunkard, Harris, Pedersen, \& McClearn, 1990; Wake et al., 2007; Whitaker, Wright, Pepe, Seidel, \& Dietz, 1997). Our findings differ from a study that assessed relationships between BMI and parenting styles on 4- and 5-year-old children. Within that study, it was found that the parental BMI status was the strongest predictor and that the relationship was not attenuated by inclusion of parenting styles or dimension (Rhee et al., 2006; Wake et al., 2007).

In congruence with the conceptual framework, an increased level of authoritarian feeding (restriction and monitoring of food) and parenting behaviors (restriction) were found when the parent expressed concern with their child's weight. A longitudinal design would be required to determine whether these behaviors were present before the concern or whether they were accentuated in the presence of concern with the child's weight. The parenting (restriction) and feeding behaviors (restriction of food and pressure to eat) were highly predictive of the child's BMI\% in this cross-sectional study.

Further research needs to be performed that examines how a parent's behavior related to nutrition influences a child and this research needs to include diverse samples with children of different age groups. It will be important to further understand how different ethnic groups respond over time to having a concern about their child's weight. The underlying assumption that parents want to help their children and will be attentive to suggestions if they believe their child is at risk for harm should be followed. It is vital for health care providers to work with families to educate them when there is a viable concern related to the child's weight. Simultaneously, providers need to ascertain that a concern regarding the child's weight is accurate and educate families on where their child's BMI places them with regards to weight status and health risks.

When working with African American families, acknowledgment of increased use of authoritarian parenting and feeding behaviors should be noted. Understanding that these practices may be cultural or related to the environment that the family lives in should be taken into account. Taking time to identify whether the parents are concerned and questioning what feeding and parenting practices are being used may open the discussion on how authoritarian behaviors may negatively be impacting their child's weight status.

When the concern is present, health care providers can take the opportunity to teach appropriate approaches for parents to use to improve the child's health habits. In the optimal situation, feeding behaviors and general parenting behaviors should be assessed early on before the behaviors become engrained and feeding habits are entrenched in the child. When that has not been done, providers need to assess parenting and feeding behaviors at later ages, acknowledging that children are still receptive to their parents and amenable to change. When dealing with an older child, it may be beneficial to include the child or adolescent in the discussion about what feeding behaviors are being used. Education directly with the child can help them make better choices (Foster et al., 2010). 
Integrating the family's culture into care will be necessary as it is clear that there are differences between ethnic groups.

Because of the cross-sectional design of this study, causality is unable to be determined. Because the parenting and feeding behaviors were assessed at a single meeting, it is unknown whether the parents had used different parenting or feeding practices before they expressed concern about their child's weight. Additional limitations include that the findings cannot be generalized to other age groups or ethnic groups and the use of self-report opens the possibility of participants providing the socially desirable answer to questions.

In the attempts to reduce or curb the pediatric obesity epidemic, it would seem logical to continue to focus on the parent as the agent of change. Parenting behaviors and feeding behaviors have a direct impact on a child's weight. Providers should be aware of this relationship and attempt to teach parents of children of all ages appropriate feeding behaviors. The second priority is to assist parents in becoming aware of how their parenting style and concerns with weight may impact their child's weight status. Interventions to help parents develop authoritative parenting and feeding behaviors with older children need to be developed and tested.

\section{Declaration of Conflicting Interests}

The authors declared that they had no conflicts of interest with respect to their authorship or the publication of this article.

\section{Funding}

The authors received the following financial support for the research and/or authorship of this article: Financial support provided through the Consortium for Pediatric Nursing Research Development Grant funded by Children's Research Institute of Children's Hospital of Wisconsin.

\section{References}

Barber, B., Stolz, H., Olsen, J. (2005). Parental support, psychological control and behavioral control: Assessing relevance across time, method and culture. Monographs of the Society for Research in Child Development, 70, 1-136.

Baughcum, A., Chamberlin, L., Deeks, C., Powers, S., Whitaker, R. (2000). Maternal perceptions of overweight children. Pediatrics, 106, 1380-1386.

Baumrind, D. (1966). Effects of authoritative parental control on child behavior. Child Development, 37, 886-907.

Baumrind, D. (2005). Patterns of parental authority and adolescent autonomy. New Directions for Child and Adolescent Development, 108, 61-69.

Birch, L., Fisher, J. (2000). Mothers' child-feeding practices influence daughters' eating and weight. American Journal of Clinical Nutrition, 71, 1054-1061.

Birch, L., Fisher, J., Grimm-Thomas, K., Markey, C., Sawyer, R., Johnson, S. (2001). Confirmatory factor analysis of the child feeding questionnaire: A measure of parental attitudes, beliefs and practices about child feeding and obesity proneness. Appetite, 36, 201-210.

Butler, J., Skinner, M., Gelfand, D., Berg, C., Wiebe, D. (2007). Maternal parenting style and adjustment in adolescents with type I diabetes. Journal of Pediatric Psychology, 32, 12271237. 
Centers for Disease Control and Prevention . (2009). Obesity prevalence among low-income, preschool-aged children-United States, 1998-2008. Morbidity and Mortality Weekly Report, $58,769-773$.

Costanzo, P., Woody, E. (1985). Domain-specific parenting styles and their impact on the child's development of particular deviance: The example of obesity proneness. Journal of Social and Clinical Psychology, 3, 425-445.

Demory-Luce, D., Morales, M., Nicklas, T., Baranowski, T., Zakeri, I., Berenson, G. (2004). Changes in food group consumption patterns from childhood to young adulthood: The Bogalusa heart study. Journal of the American Dietetic Association, 104, 1683-1691.

Doolen, J., Alpert, P., Miller, S. (2009). Parental disconnect between perceived and actual weight status of children: A metasynthesis of the current literature. Journal of the American Academy of Nurse Practitioners, 21, 160-166.

Dornbusch, S., Ritter, P., Leiderman, P., Roberts, D., Fraleigh, M. (1987). The relation of parenting style to adolescent school performance. Child Development, 58, 1244-1257.

Eckstein, K., Mikhail, L., Ariza, A., Thomson, S., Millard, S., Binns, H. (2006). Parents' perceptions of their child's weight and health. Pediatrics, 117, 681-690.

Eneli, I., Crum, P., Tylka, T. (2008). The trust model: A different feeding paradigm for managing childhood obesity. Obesity, 16, 2197-2204.

Fisher, J., Birch, L. (1999). Restricting access to palatable foods affects children's behavioral response, food selection and intake. American Journal of Clinical Nutrition, 69, 1264-1272.

Foster, G., Linder, B., Baranowski, T., Cooper, D., Goldberg, L., Harrell, J., . . . Hirst, K. (2010). A school-based intervention for diabetes risk reduction. New England Journal of Medicine, 363, 443-453.

Golan, M., Crow, S. (2004). Targeting parents exclusively in the treatment of childhood obesity: Longterm results. Obesity Research, 12, 357-361.

Golan, M., Kaufman, V., Shahar, D. (2006). Childhood obesity treatment: Targeting parents exclusively v. parents and children. British Journal of Nutrition, 95, 1008-1015.

Goodman, E., Hinden, B., Khandelwai, S. (2000). Accuracy of teen and parental reports of obesity and body mass index. Pediatrics, 106, 52-58.

Greenley, R., Holmbeck, G., Rose, B. (2006). Predictors of parenting behavior trajectories among families of young adolescents with and without spina bifida. Journal of Pediatric Psychology, 31, 1057-1071.

Hughes, S., Power, T., Orlet Fisher, J., Mueller, S., Nicklas, T. (2005). Revisiting a neglected construct: Parenting styles in a child-feeding context. Appetite, 44, 83-92.

Kaur, H., Li, C., Nazir, N., Choi, W., Resnicow, K., Birch, L., Ahluwalia, J. S. (2006). Confirmatory factor analysis of the child-feeding questionnaire among parents of adolescents. Appetite, 47, 3645.

Klesges, R., Stein, R., Eck, L., Isbell, T., Klesges, L. (1991). Parental influence of food selection in young children and its relationships to childhood obesity. American Journal of Clinical Nutrition, 53, 859-864.

Luther, B. (2007). Looking at childhood obesity through the lens of Baumrind's parenting typologies. Orthopaedic Nursing, 26, 270-278.

Maccoby, E., Martin, J. (1983). Handbook of child psychology: Volume 4. Socialization, personality, and social development (4th ed.). New York, NY: John Wiley.

Maynard, L., Galuska, D., Blanck, H., Serdula, M. (2003). Maternal perceptions of weight status of children. Pediatrics, 111, 1226-1231. 
Moens, E., Braet, C., Soetens, B. (2007). Observation of family functioning at mealtime: A comparison between families of children with and without overweight. Journal of Pediatric Psychology, 32, 52-63.

Musher-Eizenman, D., Holub, S., Hauser, J., Young, K. (2007). The relationship between parents' antifat attitudes and restrictive feeding. Obesity, 15, 2095-2102.

Ogden, C., Carroll, M., Curtin, L., Lamb, M., Flegal, K. (2010). Prevalence of high body mass index in US children and adolescents, 2007-2008. Journal of the American Medical Association, 303, 242-249.

O’Neil, M., Shewokis, P., Falkenstein, K., DeLago, C., Smith, S., Vaughn, N., Costigan, T. E. (2010). Psychosocial factors and health perceptions in parents and children who are overweight or obese. Obesity, 18, 1558-1565.

Polit, D., Beck, C. (2008). Nursing research: Generating and assessing evidence for nursing practice (8th ed.). Philadelphia, PA: Lippincott Williams \& Wilkins.

Rhee, K., Lumeng, J., Appugliese, D., Kaciroti, N., Bradley, R. (2006). Parenting styles and overweight status in first grade. Pediatrics, 117, 2047-2054.

Rudy, E., Estok, P., Kerr, M., Menzel, L. (1994). Research incentives: Money versus gifts. Nursing Research, 43, 253-255.

Savage, J., Fisher, J., Birch, L. (2007). Parental influence on eating behavior: Conception to adolescence. Journal of Law, Medicine \& Ethics, 35, 22-34.

Schaefer, E. (1965). Children's report on parental behavior: An inventory. Child Development, 36, 413-424.

Schludermann, S., Schludermann, E. (1988). Questionnaire for children and youth (CRPBI-30). Unpublished manuscript, University of Manitoba, Winnipeg, Canada.

Singh, G., Siahpush, M., Kogan, M. (2010). Rising social inequalities in US childhood obesity, 20032007. Annals of Epidemiology, 20, 40-52.

Steinberg, L., Lamborn, S., Darling, N., Mounts, N., Dornbusch, S. (1994). Over time changes in adjustment and competence among adolescents from authoritative, indulgent, and neglectful families. Child Development, 65, 754-770.

Steinke, E. (2004). Research ethics, informed consent, and participation recruitment. Clinical Nurse Specialist, 18, 88-95.

Stunkard, A., Harris, J., Pedersen, N., McClearn, G. (1990). The body mass index of twins who have been reared apart. New England Journal of Medicine, 322, 1483-1487.

Van der Horst, K., Oenema, A., Ferreira, I., Wendel-Vos, W., Giskes, K., Van Lenthe, F., Brug, J. (2007). A systematic review of environmental correlates of obesity-related dietary behaviors in youth. Health Education Research, 22, 203-226.

Wake, M., Nicholson, J., Hardy, P., Smith, K. (2007). Preschooler obesity and parenting styles of mothers and fathers: Australian national population study. Pediatrics, 120, e1520-e1527.

Wang, Y., Beydoun, M. (2007). The obesity epidemic in the United States-Gender, age, socioeconomic, racial/ethnic and geographic characteristics: A systematic review and metaregression analysis. Epidemiologic Reviews, 29, 6-28.

Wardle, J., Carnell, S. (2006). Parental feeding practices and children's weight. Acta Paediatrica, 96(Suppl. s454), 5-11.

Whitaker, R., Wright, J., Pepe, M., Seidel, K., Dietz, W. (1997). Predicting obesity in young adulthood from childhood and parental obesity. New England Journal of Medicine, 337, 869-873. 
Young-Hyman, D., Herman, L., Scott, D., Schlundt, D. (2000). Caregiver perception of children's obesity-related health risk: A study of African American families. Obesity Research, 8, 241248. 